\title{
Infiltrative coating
}

Energy Environ. Sci. https://doi.org/10.1039/

DOEE01221A (2020)

Grid-scale storage technologies demand cheaper and safer alternatives to Li-ion batteries. Aqueous $\mathrm{Zn}$-ion batteries have received increasing interest due to their non-flammable aqueous electrolytes and the elemental abundance of $\mathrm{Zn}$. One approach is the $\mathrm{Ni}-\mathrm{Zn}$ type battery that typically consists of an oxyhydroxide cathode, an alkaline electrolyte and a $\mathrm{Zn}$ anode. However, research efforts so far have not been able to achieve the high mass loading of cathode materials $\left(35 \mathrm{mAh} \mathrm{cm}^{-2}\right)$ required for industrial applications. Consequently, the battery energy density is far below its theoretical value. Now, Dongliang Chao, Yungui Chen and colleagues from China and Australia report a cathode made of NiS-coated metal hydroxides that enables a Ni-Zn battery to achieve a high energy density of $165 \mathrm{Wh} \mathrm{kg}^{-1}$ (506 Wh l-1), which is competitive for grid applications.

In their fabrication, $\mathrm{Ni}_{0.95} \mathrm{Zn}_{0.05}(\mathrm{OH})_{2.0}$ microspheres are first prepared via a precipitation method, and subsequently immersed into a $\mathrm{Na}_{2} \mathrm{~S}$ solution to allow the formation of thin NiS coating layers. The inner spherical structure is very compact so that high mass loadings (up to $41 \mathrm{mAh} \mathrm{cm}^{-2}$ ) are achieved. Importantly, both the $\mathrm{S}^{2-}$ ions and metal ions diffuse across the interface between the inner structure and the coating, creating abundant and interconnected mesopores. This increases the surface area and enhances the electrochemical performance of the cathode. To ensure the high-performance of the $\mathrm{Ni}-\mathrm{Zn}$ battery, the researchers also optimize the anode structure to suppress $\mathrm{Zn}$ dendrite formation and reformulate the electrolyte to prevent the corrosion of the anode.

Changjun Zhang

Published online: 17 July 2020

https://doi.org/10.1038/s41560-020-0658-x 